\title{
Finite-time nonautonomous bifurcation in impulsive systems
}

\author{
Marat Akhmet and Ardak Kashkynbayev ${ }^{\bowtie}$ \\ Department of Mathematics, Middle East Technical University, 06800 Ankara, Turkey \\ Appeared 11 August 2016 \\ Communicated by Tibor Krisztin
}

\begin{abstract}
The purpose of this article is to investigate nonautonomous bifurcation in impulsive differential equations. The impulsive finite-time analogues of transcritical and pitchfork bifurcation are provided. An illustrative example is given with numerical simulations which support theoretical results.
\end{abstract}

Keywords: nonautonomous bifurcation theory, impulsive differential equations, finitetime dynamics, pitchfork bifurcation, transcritical bifurcation.

2010 Mathematics Subject Classification: 34A34, 34A37, 34C23, 34D05, $37 \mathrm{~B} 55$.

\section{Introduction}

The fields of attractivity and bifurcation are related subjects, because a bifurcation is often associated with loss or gain of attractivity. For continuous dynamical systems there are qualitative papers devoted to nonautonomous bifurcation theory studied in the last twenty years $[8,9,11,12,14-16]$. However, from applications viewpoint one is interested in the behavior of the system on finite time interval. The need to analyze such equations arises in many applications such as transport problems in fluid, ocean or atmosphere dynamics [13], and also increasingly in biological applications [6,17]. There are at least two reasons why one is interested in dynamics on bounded time-sets. The first reason is the interest in transient behavior of solutions although the differential equation might be given on the real half-line. Another reason is the simple fact that the data deduced from observations or measurements is mostly given only on a bounded time-set.

Many evolutionary processes in the real world are characterized by sudden changes at certain times. These changes are called to be impulsive phenomena $[1,7,10,18]$, which are widespread in modeling in mechanics, electronics, biology, neural networks, medicine, and social sciences $[1,2,5]$. An impulsive differential equation is one of the basic instruments to understand the role of discontinuity better for the real world problems. Extending nonautonomous bifurcation theory to impulsive systems is a contemporary problem. In the papers $[3,4]$ we have studied nonautonomous bifurcation in impulsive systems without finite-time

\footnotetext{
${ }^{\bowtie}$ Corresponding author. Email: ardaky@gmail.com
} 
viewpoint. The main novelty of this paper is to provide suitable and efficient concepts of finite-time bifurcation in the context of nonautonomous differential equations with impulses.

This paper is organized as follows. In Section 2 we give basic definitions and definitions of finite-time attractors and repellers. In Section 3 the results of linearized attractivity and repulsivity are presented. Section 4 is devoted to impulsive finite-time analogues of nonautonomous transcritical and pitchfork bifurcations respectively. Finally, in Section 5 we give an example which supports our theoretical discussion.

\section{Preliminaries}

We denote by $\mathbb{R}$ the set of all real numbers, $\mathbb{Z}$ the set of integers. In this section we introduce concepts of attractive and repulsive solutions, which are used to analyze asymptotic behavior of impulsive non-autonomous systems. This paper is concerned with systems of the type

$$
\begin{aligned}
\dot{x} & =f(t, x), \\
\left.\Delta x\right|_{t=\theta_{i}} & =J_{i}(x),
\end{aligned}
$$

where $\left.\Delta x\right|_{t=\theta_{i}}:=x\left(\theta_{i}+\right)-x\left(\theta_{i}\right), x\left(\theta_{i}+\right)=\lim _{t \rightarrow \theta_{i}^{+}} x(t)$. The system (2.1) is defined on the set $\Omega=I \times \mathbb{A} \times G$ where $G \subseteq \mathbb{R}^{n}, I \subset \mathbb{R}$ is a finite compact time interval which contains only a finite number of impulse points $\theta_{i}$ with the set of indexes $\mathbb{A}$. Let $\phi\left(t, t_{0}, x_{0}\right)$ be general solution of (2.1) which is uniquely determined and non-continuable.

Let $P C(\mathbb{R}, \theta)$ denote the space of piecewise left continuous functions with discontinuity of the first kind at points in the sequence $\theta$. The Euclidean space $\mathbb{R}^{n}$ is equipped with the norm $\|\cdot\|$, and write $B_{\epsilon}\left(x_{0}\right)=\left\{x \in \mathbb{R}^{n}:\left\|x-x_{0}\right\|<\epsilon\right\}$ for arbitrary $\epsilon$-neighborhood of some point $x_{0} \in \mathbb{R}^{n}$. We use Hausdorff semi-distance between nonempty set $A$ and $B$ as $d(A, B)=$ $\sup _{a \in A} \inf _{b \in B} d(a, b)$. For arbitrary nonempty set $X \subset \mathbb{R}^{n}$ define $\phi\left(t, t_{0}, X\right):=\bigcup_{x_{0} \in X} \phi\left(t, t_{0}, x_{0}\right)$.

A set $M \subset I \times \mathbb{R}^{n}$ is called nonautonomous set if for all $t \in I$, $t$-fibers $M(t):=\left\{x \in \mathbb{R}^{n}\right.$ : $(t, x) \in M\}$ are nonempty. $M$ is said to be compact if all $t$-fibers are compact. $M$ is said to be invariant if $\phi\left(t, t_{0}, M\left(t_{0}\right)\right)=M(t)$ for all $t, t_{0} \in I$.

Let $f: X \rightarrow Y$ be a given function. The graph of $f$ is defined by

$$
\operatorname{graph} f:=\{(x, y) \in X \times Y: y=f(x)\} \text {. }
$$

Asymptotic properties of continuous dynamics and dynamics with discontinuous are the same. In what follows we use definitions of attractivity and repulsivity without any changes form [16].

Definition 2.1. Let $t_{0} \in I$ and $T>0$ with $t_{0}+T \in I, A$ and $R$ be compact and invariant nonautonomous sets.

- $A$ is called $\left(t_{0}, T\right)$-attractor if

$$
\limsup _{\epsilon \searrow 0} \frac{1}{\epsilon} d\left(\phi\left(t_{0}+T, t_{0}, B_{\epsilon}\left(A\left(t_{0}\right)\right)\right), A\left(t_{0}+T\right)\right)<1 .
$$

- A solution $\psi:\left[t_{0}, t_{0}+T\right] \rightarrow \mathbb{R}^{n}$ of $(2.1)$ is called $\left(t_{0}, T\right)$-attractive if graph $\psi$ is a $\left(t_{0}, T\right)$ attractor. 
- $R$ is called $\left(t_{0}, T\right)$-repeller if

$$
\limsup _{\epsilon \searrow 0} \frac{1}{\epsilon} d\left(\phi\left(t_{0}+T, t_{0}, B_{\epsilon}\left(R\left(t_{0}+T\right)\right)\right), R\left(t_{0}\right)\right)<1 .
$$

- A solution $\psi:\left[t_{0}, t_{0}+T\right] \rightarrow \mathbb{R}^{n}$ of (2.1) is called $\left(t_{0}, T\right)$-repulsive if graph $\psi$ is a $\left(t_{0}, T\right)$ repeller.

Note that the notions of finite-time attractivity and repulsivity are not invariant with respect to a change of the metric $d$ to an equivalent metric. Moreover, the notions of $\left(t_{0}, T\right)$ attractor and $\left(t_{0}, T\right)$-repeller satisfy duality principle in the sense that they change their roles under time reversal.

Example 2.2. Let $I:=\left[t_{0}, t_{0}+T\right]$ be an interval containing a finite number of impulse points $\theta_{i}$ such that $t_{0} \leq \theta_{1}<\theta_{2}<\cdots<\theta_{m} \leq t_{0}+T$ for some $t_{0} \in \mathbb{R}, T>0$ and $m \in \mathbb{N}$. Consider the system

$$
\begin{aligned}
\dot{x} & =a(t) x, \\
\left.\Delta x\right|_{t=\theta_{i}} & =b_{i} x,
\end{aligned}
$$

with piecewise continuous function $a: I \rightarrow \mathbb{R}$ and there exist constants $b, B \in \mathbb{R}$ such that $-1<b \leq b_{i} \leq B$. Let $\Phi: I \times I \rightarrow \mathbb{R}^{n}$ be the transition matrix of the system (2.1).

If $t_{0}<\theta_{1}$, then $a(t)$ is continuous on $\left[t_{0}, \theta_{1}\right]$ since $a(t) \in P C(\mathbb{R}, \theta)$. So, we have that $\Phi\left(\theta_{1}, t_{0}\right)=\exp \left(\int_{t_{0}}^{\theta_{1}} a(s) d s\right)$. At $t=\theta_{1}$ the solution makes a jump and we have that $x\left(\theta_{1}+\right)=\left(1+b_{1}\right) x\left(\theta_{1}\right)$. Next, $a(t)$ being continuous on $\left(\theta_{1}, \theta_{2}\right]$ implies that $\Phi\left(\theta_{2}, \theta_{1}\right)=$ $\exp \left(\int_{\theta_{1}}^{\theta_{2}} a(s) d s\right)\left(1+b_{1}\right)$. Proceeding in this way one can show that

$$
\Phi\left(t_{0}+T, t_{0}\right)=\Phi\left(\theta_{1}, t_{0}\right) \Phi\left(\theta_{2}, \theta_{1}\right) \cdots \Phi\left(t_{0}+T, \theta_{m}\right)=e^{\int_{t_{0}}^{t_{0}+T} a(s) d s} \prod_{i=1}^{m}\left(1+b_{i}\right)
$$

since $1+b_{i}$ is nonsingular matrix and commutes with any other matrix because $1+b_{i}>0$.

If $t_{0}=\theta_{1}$, then the solution starts with a jump and we have that $x\left(\theta_{1}+\right)=\left(1+b_{1}\right) x\left(\theta_{1}\right)$. Next, $a(t)$ is continuous on $\left(\theta_{1}, \theta_{2}\right]$ implies that $\Phi\left(\theta_{2}, \theta_{1}\right)=\exp \left(\int_{\theta_{1}}^{\theta_{2}} a(s) d s\right)\left(1+b_{1}\right)$. Arguing this way one can show that

$$
\Phi\left(t_{0}+T, t_{0}\right)=\Phi\left(\theta_{2}, \theta_{1}\right) \cdots \Phi\left(t_{0}+T, \theta_{m}\right)=e^{\int_{t_{0}}^{t_{0}+T} a(s) d s} \prod_{i=1}^{m}\left(1+b_{i}\right) .
$$

We want to point out that the basics of linear impulsive systems are fruitfully discussed in the books $[1,7,18]$. As a result, we have that

$$
\begin{aligned}
\Phi\left(t_{0}+T, t_{0}\right)=e^{\int_{t_{0}}^{t_{0}+T} a(s) d s} \prod_{i=1}^{m}\left(1+b_{i}\right) & \leq e^{\int_{t_{0}}^{t_{0}+T} a(s) d s} \prod_{i=1}^{m}(1+B) \\
& =e^{\int_{t_{0}}^{t_{0}+T} a(s) d s+m \ln (1+B)} .
\end{aligned}
$$

Therefore, any invariant and compact nonautonomous set is a $\left(t_{0}, T\right)$-attractor if

$$
\int_{t_{0}}^{t_{0}+T} a(s) d s+m \ln (1+B)<0 .
$$

By the same way one can say that any invariant and compact nonautonomous set is a $\left(t_{0}, T\right)$ repeller if $\int_{t_{0}}^{t_{0}+T} a(s) d s+m \ln (1+b)>0$. 
Definition 2.3. The radius of $\left(t_{0}, T\right)$-attraction of a $\left(t_{0}, T\right)$-attractor $A$ is defined by

$$
\mathcal{A}_{A}^{\left(t_{0}, T\right)}:=\sup \left\{\epsilon>0: d\left(\phi\left(t_{0}+T, t_{0}, B_{\widehat{\epsilon}}\left(A\left(t_{0}\right)\right)\right), A\left(t_{0}+T\right)\right)<\widehat{\epsilon} \text { for all } \widehat{\epsilon} \in(0, \epsilon)\right\},
$$

and the radius of $\left(t_{0}, T\right)$-repulsion of a $\left(t_{0}, T\right)$-repeller $R$ is defined by

$$
\mathcal{R}_{R}^{\left(t_{0}, T\right)}:=\sup \left\{\epsilon>0: d\left(\phi\left(t_{0}+T, t_{0}, B_{\widehat{\epsilon}}\left(R\left(t_{0}+T\right)\right)\right), R\left(t_{0}\right)\right)<\widehat{\epsilon} \text { for all } \widehat{\epsilon} \in(0, \epsilon)\right\} .
$$

In Example 2.2 every invariant and compact set $S \subset\left[t_{0}, t_{0}+T\right] \times \mathbb{R}$ of the system (2.2) is

- $\left(t_{0}, T\right)$-attractor with $\mathcal{A}_{S}^{\left(t_{0}, T\right)}=\infty$ if $\int_{t_{0}}^{t_{0}+T} a(s) d s+m \ln (1+B)<0$,

- $\left(t_{0}, T\right)$-repeller with $\mathcal{R}_{S}^{\left(t_{0}, T\right)}=\infty$ if $\int_{t_{0}}^{t_{0}+T} a(s) d s+m \ln (1+b)>0$.

Definition 2.4. We consider the impulsive system (2.1), which depends on a parameter $\mu$. For a given $\mu_{0} \in\left(\mu_{-}, \mu_{+}\right)$, we say that system (2.1) admits a supercritical $\left(t_{0}, T\right)$-bifurcation at $\mu_{0}$ if there exist a $\widehat{\mu}>\mu_{0}$ a piecewise continuous function $\psi:\left[t_{0}, t_{0}+T\right] \times\left(\mu_{0}, \widehat{\mu}\right) \rightarrow \mathbb{R}^{n}$ such that one of the following two statements is fulfilled:

- $\psi(\cdot, \mu)$ is a $\left(t_{0}, T\right)$-attractive solution of the system (2.1) for all $\mu \in\left(\mu_{0}, \widehat{\mu}\right)$, and

$$
\lim _{\mu \searrow \mu_{0}} \mathcal{A}_{\psi(\cdot, \mu)}^{\left(t_{0}, T\right)}=0
$$

- $\psi(\cdot, \mu)$ is a $\left(t_{0}, T\right)$-repulsive solution of the system (2.1) for all $\mu \in\left(\mu_{0}, \widehat{\mu}\right)$, and

$$
\lim _{\mu \searrow \mu_{0}} \mathcal{R}_{\psi(\cdot, \mu)}^{\left(t_{0}, T\right)}=0
$$

Subcritical $\left(t_{0}, T\right)$-bifurcation is defined by interchanging the limit into $\mu \nearrow \mu_{0}$.

\section{Attractivity and repulsivity in nonhomogeneous linear impulsive systems}

In this section we study linearized systems in finite-time with definitions provided in the previous section which play great role in the stability analysis of solutions of nonlinear impulsive systems with fixed moments of impulses. Let us consider the impulsive system in a compact interval $I:=\left[t_{0}, t_{0}+T\right]$ with $m$ impulse points $\theta_{i}$ for some $t_{0} \in \mathbb{R}, T>0$ and $m \in \mathbb{N}$,

$$
\begin{aligned}
\dot{x} & =A(t) x+F(t, x), \\
\left.\Delta x\right|_{t=\theta_{i}} & =B_{i} x+J_{i}(x),
\end{aligned}
$$

where $A \in P C(I, \theta)$, matrices $B_{i}$ satisfy $\operatorname{det}\left(B_{i}+I\right) \neq 0, F: I \times G \rightarrow \mathbb{R}^{n}$ and $J: \mathbb{A} \times G \rightarrow \mathbb{R}^{n}$. Denote $\phi\left(t, t_{0}, x_{0}\right)$ as the solution of (3.1) and $\Phi: I \times I \rightarrow \mathbb{R}^{n \times n}$ as the transition matrix of the linearized system

$$
\begin{aligned}
\dot{x} & =A(t) x, \\
\left.\Delta x\right|_{t=\theta_{i}} & =B_{i} x .
\end{aligned}
$$

Define

$$
M_{+}:=\sup \left\{\|\Phi(t, s)\|: t_{0} \leq s \leq t \leq t_{0}+T\right\}
$$


and

$$
M_{-}:=\sup \left\{\|\Phi(t, s)\|: t_{0} \leq t \leq s \leq t_{0}+T\right\}
$$

Assume that the following conditions hold for the system (3.1):

(C1) $\left\|\Phi\left(t_{0}+T, t_{0}\right)\right\|<1$;

(C2) the functions $F(t, x)$ and $J_{i}(x)$ are Lipschitzian i.e., $\|F(t, x)\| \leq l\|x\|,\left\|J_{i}(x)\right\| \leq l\|x\|$ for all $t \in I, i \in \mathbb{A}$ and $\|x\|<h, h>0$.

Then one has the following theorem.

Theorem 3.1. The trivial solution of the system (3.1) is $\left(t_{0}, T\right)$-attractive for sufficiently small values of l, i.e.,

$$
\left\|\phi\left(t_{0}+T, t_{0}, x_{0}\right)\right\| \leq \delta e^{M_{+} T l+m \ln \left(1+M_{+} l\right)+\ln \left\|\Phi\left(t_{0}+T, t_{0}\right)\right\|} .
$$

Now consider the following condition

(C3) $\left\|\Phi\left(t_{0}, t_{0}+T\right)\right\|<1$.

Theorem 3.2. Assume that conditions (C2) and (C3) are true for the system (3.1), then the trivial solution of (3.1) is $\left(t_{0}, T\right)$-repulsive for sufficiently small values of $l$, i.e.,

$$
\left\|\phi\left(t_{0}, t_{0}+T, x_{0}\right)\right\| \leq \delta e^{M-T l+m \ln \left(1+M_{-} l\right)+\ln \left\|\Phi\left(t_{0}, t_{0}+T\right)\right\|} .
$$

Proof. We prove only Theorem 3.1 since Theorem 3.2 can be proven analogously. An equivalent integral equation of the system (3.1) can be written as [1,18]:

$$
\phi\left(t, t_{0}, x_{0}\right)=\Phi\left(t, t_{0}\right) x_{0}+\int_{t_{0}}^{t} \Phi(t, s) F\left(s, \phi\left(s, t_{0}, x_{0}\right)\right) d s+\sum_{t_{0} \leq \theta_{i}<t} \Phi\left(t, \theta_{i}\right) I_{i}\left(\phi\left(\theta_{i}, t_{0}, x_{0}\right)\right.
$$

for all $t \in I$. Therefore, we get

$$
\left\|\phi\left(t, t_{0}, x_{0}\right)\right\| \leq\left\|\Phi\left(t, t_{0}\right)\right\|\left\|x_{0}\right\|+M_{+} l \int_{t_{0}}^{t}\left\|\phi\left(s, t_{0}, x_{0}\right)\right\| d s+M_{+} l \sum_{t_{0} \leq \theta_{i}<t}\left\|\phi\left(\theta_{i}, t_{0}, x_{0}\right)\right\|
$$

for all $t \in I$ is fulfilled. Hence, by the Gronwall-Bellman lemma for piecewise continuous functions $[1,18]$ it follows that

$$
\begin{aligned}
\left\|\phi\left(t_{0}+T, t_{0}, x_{0}\right)\right\| & \leq\left\|\Phi\left(t_{0}+T, t_{0}\right)\right\| e^{M_{+} l T}\left(1+M_{+} l\right)^{i\left[t_{0}, t_{0}+T\right)}\left\|x_{0}\right\| \\
& \leq\left\|x_{0}\right\| e^{\ln \left\|\Phi\left(t_{0}+T, t_{0}\right)\right\|+M_{+} l T+m \ln \left(1+M_{+} l\right)}
\end{aligned}
$$

where $i\left[t_{0}, t_{0}+T\right)$ is the number of elements of the sequence $\theta_{i}$ in the interval $\left[t_{0}, t_{0}+T\right)$. Since in this paper $i\left[t_{0}, t_{0}+T\right)=m$, one can see that the required result follows by choosing $\delta=K h$ for $l$ small enough that $\ln \left\|\Phi\left(t_{0}+T, t_{0}\right)\right\|+M_{+} l T+m \ln \left(1+M_{+} l\right)<0$.

\section{Bifurcation analysis}

In this section we state and prove finite-time nonautonomous transcritical and pitchfork bifurcation results for impulsive systems. In what follows, the auxiliary theorems obtained in the previous section for higher dimensions will be used in the scalar case. 


\subsection{The transcritical bifurcation}

In this subsection we study impulsive analogue of the nonautonomous transcritical bifurcation in finite-time. Let $x_{-}<0<x_{+}$and $\mu_{-}<\mu_{+}$be real numbers and let $I:=\left[t_{0}, t_{0}+T\right]$ with $m$ impulse points $\theta_{i}$. Consider the system

$$
\begin{aligned}
\dot{x} & =p(t, \mu) x+q(t, \mu) x^{2}+r(t, x, \mu), \\
\left.\Delta x\right|_{t=\theta_{i}} & =a_{i}(\mu) x+b_{i}(\mu) x^{2}+c_{i}(x, \mu),
\end{aligned}
$$

with piecewise continuous functions $p: I \times\left(\mu_{-}, \mu_{+}\right) \rightarrow \mathbb{R}, q: I \times\left(\mu_{-}, \mu_{+}\right) \rightarrow \mathbb{R}$ and $r: I \times$ $\left(x_{-}, x_{+}\right) \times\left(\mu_{-}, \mu_{+}\right) \rightarrow \mathbb{R}$ satisfying $r(t, 0, \alpha)=0, a: \mathbb{A} \times\left(\mu_{-}, \mu_{+}\right) \rightarrow \mathbb{R}, b: \mathbb{A} \times\left(\mu_{-}, \mu_{+}\right) \rightarrow \mathbb{R}$ and $c: \mathbb{A} \times\left(x_{-}, x_{+}\right) \times\left(\mu_{-}, \mu_{+}\right) \rightarrow \mathbb{R}$ with $a_{i}(\mu) \neq-1$ and $c_{i}(0, \mu)=0$. Let $\Phi_{\mu}(t, s)$ be the fundamental matrix of the associated homogeneous part of the system

$$
\begin{aligned}
\dot{x} & =p(t, \mu) x, \\
\left.\Delta x\right|_{t=\theta_{i}} & =a_{i}(\mu) x .
\end{aligned}
$$

Assume that there exists $\mu_{0} \in\left(\mu_{-}, \mu_{+}\right)$such that the following conditions hold:

(T1) $\Phi_{\mu}\left(t_{0}+T, t_{0}\right)<1$ for all $\mu \in\left(\mu_{-}, \mu_{0}\right)$ and $\Phi_{\mu}\left(t_{0}+T, t_{0}\right)>1$ for all $\mu \in\left(\mu_{0}, \mu_{+}\right)$;

or

(T1*) $\Phi_{\mu}\left(t_{0}+T, t_{0}\right)>1$ for all $\mu \in\left(\mu_{-}, \mu_{0}\right)$ and $\Phi_{\mu}\left(t_{0}+T, t_{0}\right)<1$ for all $\mu \in\left(\mu_{0}, \mu_{+}\right)$.

The quadratic terms either fulfill:

(T2) $\liminf _{\mu \rightarrow \mu_{0}} \inf _{t \in I} q(t, \mu)>0$ and $\liminf _{\mu \rightarrow \mu_{0}} \inf _{i \in \mathbb{A}} b_{i}(\mu)>0$;

or

(T2*) $\limsup _{\mu \rightarrow \mu_{0}} \sup _{t \in I} q(t, \mu)<0$ and $\lim \sup _{\mu \rightarrow \mu_{0}} \sup _{i \in \mathbb{A}} b_{i}(\mu)<0$.

And the remainders satisfy:

(T3) $\lim _{x \rightarrow 0} \sup _{\mu \in\left(\mu_{0}-|x|, \mu_{0}+|x|\right)} \sup _{t \in I} \frac{|r(t, x, \mu)|}{|x|^{2}}=0$;

(T4) $\lim _{x \rightarrow 0} \sup _{\mu \in\left(\mu_{0}-|x|, \mu_{0}+|x|\right)} \sup _{i \in \mathbb{A}} \frac{\left|c_{i}(x, \mu)\right|}{|x|^{2}}=0$;

(T5) there exists sufficiently small $l>0$ such that $|r(t, x, \mu)|<l|x|,\left|c_{i}(x, \mu)\right|<l|x|$ for all $\mu \in\left(\mu_{-}, \mu_{+}\right), t \in I, i \in \mathbb{A}$ and $x \in\left(x_{-}, x_{+}\right)$.

Theorem 4.1. Assume that the above conditions hold for the system (4.1). Then there exist $\widehat{\mu}_{-}<$ $0<\widehat{\mu}_{+}$such that if (T1) is satisfied, then the trivial solution is $\left(t_{0}, T\right)$-attractive for $\mu \in\left(\widehat{\mu}_{-}, \mu_{0}\right)$ and $\left(t_{0}, T\right)$-repulsive for $\mu \in\left(\mu_{0}, \widehat{\mu}_{+}\right)$. The system (4.1) admits a $\left(t_{0}, T\right)$-bifurcation, since the corresponding radii of $\left(t_{0}, T\right)$-attraction and $\left(t_{0}, T\right)$-repulsion satisfy

$$
\lim _{\mu \nearrow \mu_{0}} \mathcal{A}_{0}^{\mu}=0 \text { and } \lim _{\mu \searrow \mu_{0}} \mathcal{R}_{0}^{\mu}=0 .
$$

In case $\left(T 1^{*}\right)$ is satisfied, the trivial solution is $\left(t_{0}, T\right)$-repulsive for $\mu \in\left(\widehat{\mu}_{-}, \mu_{0}\right)$ and $\left(t_{0}, T\right)$-attractive for $\mu \in\left(\mu_{0}, \widehat{\mu}_{+}\right)$. The system (4.1) admits a $\left(t_{0}, T\right)$-bifurcation, since the corresponding radii of $\left(t_{0}, T\right)$-attraction and $\left(t_{0}, T\right)$-repulsion satisfy

$$
\lim _{\mu \searrow \mu_{0}} \mathcal{A}_{0}^{\mu}=0 \quad \text { and } \quad \lim _{\mu \nearrow \mu_{0}} \mathcal{R}_{0}^{\mu}=0
$$


Proof. We give the first part of the proof since second part can be proven in the similar manner. That is, (T1) is assumed. Let $\phi_{\mu}$ be the general solution of the system (4.1). We may consider the case (T2). Choose $\widehat{\mu}_{-}<\mu_{0}<\widehat{\mu}_{+}$such that

$$
0<\inf _{\mu \in\left(\widehat{\mu}_{-}, \widehat{\mu}_{+}\right), t \in I} q(t, \mu) \quad \text { and } \quad 0<\inf _{\mu \in\left(\widehat{\mu}_{-}, \widehat{\mu}_{+}\right), i \in \mathbb{A}} b_{i}(\mu)
$$

By means of (T4) and (4.5) one can see that Theorem 3.1 and Theorem 3.2 can be applied. Thus, we get attractivity and repulsivity of the trivial solution as it was required to show in the theorem. We define $K:=\inf \left\{\Phi_{\mu}(t, s): t, s \in I, \mu \in\left(\widehat{\mu}_{-}, \mu_{0}\right)\right\} \in(0,1)$. To prove relations (4.3) and (4.4) we assume to the contrary that $\gamma:=\limsup _{\mu} \nearrow_{\mu_{0}} \mathcal{A}_{0}^{\mu}>0$. By means of (T3) and (4.5) one can show that there exist $\widetilde{\mu} \in\left(\widehat{\mu}_{-}, \mu_{0}\right), x_{0} \in(0, K \gamma)$ and $L>0$ such that

$$
q(t, \mu) x^{2}+r(t, x, \mu)>L \quad \text { and } \quad b_{i}(\mu) x^{2}+c_{i}(x, \mu)>L
$$

for all $t \in I, i \in \mathbb{A}, \mu \in\left(\widetilde{\mu}_{-}, \mu_{0}\right)$ and $x_{0} \in\left[K x_{0}, \frac{x_{0}}{K}\right]$. Next, fix $\widehat{\mu} \in\left(\widetilde{\mu}_{-}, \mu_{0}\right)$ such that $\mathcal{A}_{0}^{\widehat{\mu}}>x_{0}$ and

$$
\Phi_{\mu}\left(t_{0}+T, t_{0}\right) \geq 1-\frac{K L T}{x_{0}}
$$

Denote $\psi(t)=\phi_{\widehat{\mu}}\left(t, t_{0}, x_{0}\right)$. Since $\mathcal{A}_{0}^{\widehat{\mu}}>x_{0}$, we have

$$
\psi\left(t_{0}+T\right)<x_{0}
$$

Moreover, from the definition of $K$ and by means of (4.6), we get

$$
\psi\left(t_{0}+T\right) \geq K x_{0} \quad \text { for all } t \in[0, T]
$$

We study two cases.

Case 1. There exist a $t_{1} \in(0, T]$ such that $\psi\left(t_{0}+t_{1}\right)=\frac{x_{0}}{K}$. We choose $t_{1}$ maximal with this property. By means of (4.8), one can see that $\psi\left(t_{0}+T\right) \leq \frac{x_{0}}{K}$ for all $t \in\left[t_{1}, T\right]$. Next, we consider the integral equation of the system (4.1) at $t_{0}+T$ for fixed $\widehat{\mu}$ which start at point $t_{0}+t_{1}$.

$$
\begin{aligned}
\psi\left(t_{0}+T\right)= & \Phi_{\widehat{\mu}}\left(t_{0}+T, t_{0}+t_{1}\right) \frac{x_{0}}{K} \\
& +\int_{t_{0}+t_{1}}^{t_{0}+T} \Phi_{\widehat{\mu}}\left(t_{0}+T, s\right)\left(q(s, \widehat{\mu})(\psi(s))^{2}+r(s, \psi(s), \widehat{\mu})\right) d s \\
& +\sum_{t_{0}+t_{1} \leq \theta_{i}<t_{0}+T} \Phi_{\widehat{\mu}}\left(t_{0}+T, \theta_{i}\right)\left(b_{i}(\widehat{\mu})\left(\psi\left(\theta_{i}\right)\right)^{2}+c_{i}\left(\psi\left(\theta_{i}\right), \widehat{\mu}\right)\right) \\
\geq & x_{0}+K L\left(T-t_{1}\right)+\sum_{t_{0}+t_{1} \leq \theta_{i}<t_{0}+T} K L \\
> & x_{0} .
\end{aligned}
$$

This is contraction for (4.8).

Case 2. For all $t \in(0, T]$, we have $\psi\left(t_{0}+t_{1}\right)<\frac{x_{0}}{K}$. Next, from the integral equation of the 
system (4.1) at $t_{0}+T$ for fixed $\widehat{\mu}$ which start at point $t_{0}$ we have

$$
\begin{aligned}
\psi\left(t_{0}+T\right)= & \Phi_{\widehat{\mu}}\left(t_{0}+T, t_{0}+t_{1}\right) x_{0} \\
& +\int_{t_{0}}^{t_{0}+T} \Phi_{\widehat{\mu}}\left(t_{0}+T, s\right)\left(q(s, \widehat{\mu})(\psi(s))^{2}+r(s, \psi(s), \widehat{\mu})\right) d s \\
& +\sum_{t_{0} \leq \theta_{i}<t_{0}+T} \Phi_{\widehat{\mu}}\left(t_{0}+T, \theta_{i}\right)\left(b_{i}(\widehat{\mu})\left(\psi\left(\theta_{i}\right)\right)^{2}+c_{i}\left(\psi\left(\theta_{i}\right), \widehat{\mu}\right)\right) \\
\geq & \left(1-\frac{K L T}{x_{0}}\right) x_{0}+K L T+K L m \\
> & x_{0},
\end{aligned}
$$

where the last inequality follows by means of (4.6) and (4.7). But, this is again contradiction for (4.8). Hence, we have that $\lim _{\mu \nearrow \mu_{0}} \mathcal{A}_{0}^{\mu}=0$. Analogously, one can consider the condition $\left(\mathrm{T} 2^{*}\right.$ ) to show that $\lim _{\mu \searrow \mu_{0}} \mathcal{R}_{0}^{\mu}=0$.

\subsection{The pitchfork bifurcation}

In this subsection we study impulsive analogue of the nonautonomous pitchfork bifurcation. Let $x_{-}<0<x_{+}$and $\mu_{-}<\mu_{+}$be real numbers and let $I:=\left[t_{0}, t_{0}+T\right]$ with $m$ impulse points $\theta_{i}$. Consider the system

$$
\begin{aligned}
\dot{x} & =p(t, \mu) x+q(t, \mu) x^{3}+r(t, x, \mu), \\
\left.\Delta x\right|_{t=\theta_{i}} & =a_{i}(\mu) x+b_{i}(\mu) x^{3}+c_{i}(x, \mu),
\end{aligned}
$$

with piecewise continuous functions $p: I \times\left(\mu_{-}, \mu_{+}\right) \rightarrow \mathbb{R}, q: I \times\left(\mu_{-}, \mu_{+}\right) \rightarrow \mathbb{R}$ and $r: I \times$ $\left(x_{-}, x_{+}\right) \times\left(\mu_{-}, \mu_{+}\right) \rightarrow \mathbb{R}$ satisfying $r(t, 0, \alpha)=0, a: \mathbb{A} \times\left(\mu_{-}, \mu_{+}\right) \rightarrow \mathbb{R}, b: \mathbb{A} \times\left(\mu_{-}, \mu_{+}\right) \rightarrow \mathbb{R}$ and $c: \mathbb{A} \times\left(x_{-}, x_{+}\right) \times\left(\mu_{-}, \mu_{+}\right) \rightarrow \mathbb{R}$ with $a_{i}(\mu) \neq-1$ and $c_{i}(0, \mu)=0$. Let $\Phi_{\mu}(t, s)$ be the fundamental matrix of the linear system

$$
\begin{aligned}
\dot{x} & =p(t, \mu) x, \\
\left.\Delta x\right|_{t=\theta_{i}} & =a_{i}(\mu) x .
\end{aligned}
$$

Assume that there exists $\mu_{0} \in\left(\mu_{-}, \mu_{+}\right)$such that following conditions hold:

(P1) $\Phi_{\mu}\left(t_{0}+T, t_{0}\right)<1$ for all $\mu \in\left(\mu_{-}, \mu_{0}\right)$ and $\Phi_{\mu}\left(t_{0}+T, t_{0}\right)>1$ for all $\mu \in\left(\mu_{0}, \mu_{+}\right)$;

or

(P1*) $\Phi_{\mu}\left(t_{0}+T, t_{0}\right)>1$ for all $\mu \in\left(\mu_{-}, \mu_{0}\right)$ and $\Phi_{\mu}\left(t_{0}+T, t_{0}\right)<1$ for all $\mu \in\left(\mu_{0}, \mu_{+}\right)$.

The quadratic terms either fulfill:

(P2) $\liminf _{\mu \rightarrow \mu_{0}} \inf _{t \in I} q(t, \mu)>0$ and $\liminf _{\mu \rightarrow \mu_{0}} \inf _{i \in \mathbb{A}} b_{i}(\mu)>0$;

or

(P2*) $\limsup _{\mu \rightarrow \mu_{0}} \sup _{t \in I} q(t, \mu)<0$ and $\lim \sup _{\mu \rightarrow \mu_{0}} \sup _{i \in \mathbb{A}} b_{i}(\mu)<0$.

And the remainders satisfy:

(P3) $\lim _{x \rightarrow 0} \sup _{\mu \in\left(\mu_{0}-x^{2}, \mu_{0}+x^{2}\right)} \sup _{t \in I} \frac{|r(t, x, \mu)|}{|x|^{3}}=0$; 
(P4) $\lim _{x \rightarrow 0} \sup _{\mu \in\left(\mu_{0}-x^{2}, \mu_{0}+x^{2}\right)} \sup _{i \in \mathbb{A}} \frac{\left|c_{i}(x, \mu)\right|}{|x|^{3}}=0$;

(P5) there exists sufficiently small $l>0$ such that $|r(t, x, \mu)|<l|x|,\left|c_{i}(x, \mu)\right|<l|x|$ for all $\mu \in\left(\mu_{-}, \mu_{+}\right), t \in I, i \in \mathbb{A}$ and $x \in\left(x_{-}, x_{+}\right)$.

Theorem 4.2. Assume that above conditions hold for the system (4.10). Then there exist $\widehat{\mu}_{-}<0<\widehat{\mu}_{+}$ such that if the conditions (P1) and (P2) are satisfied, then the trivial solution is $\left(t_{0}, T\right)$-attractive for $\mu \in\left(\widehat{\mu}_{-}, \mu_{0}\right)$ and $\left(t_{0}, T\right)$-repulsive for $\mu \in\left(\mu_{0}, \widehat{\mu}_{+}\right)$. The system (4.10) admits a $\left(t_{0}, T\right)$-bifurcation, since the corresponding radius of $\left(t_{0}, T\right)$-attraction satisfies

$$
\lim _{\mu \nearrow \mu_{0}} \mathcal{A}_{0}^{\mu}=0
$$

If the conditions (P1) and (P2*) are satisfied, then the trivial solution is $\left(t_{0}, T\right)$-repulsive for $\mu \in$ $\left(\widehat{\mu}_{-}, \mu_{0}\right)$ and $\left(t_{0}, T\right)$-attractive for $\mu \in\left(\mu_{0}, \widehat{\mu}_{+}\right)$. The system (4.10) admits a $\left(t_{0}, T\right)$-bifurcation, since the corresponding radius of $\left(t_{0}, T\right)$-repulsion satisfies

$$
\lim _{\mu \searrow \mu_{0}} \mathcal{R}_{0}^{\mu}=0
$$

If the conditions $\left(P 1^{*}\right)$ and (P2) hold, the trivial solution is $\left(t_{0}, T\right)$-attractive for $\mu \in\left(\widehat{\mu}_{-}, \mu_{0}\right)$ and $\left(t_{0}, T\right)$-repulsive for $\mu \in\left(\mu_{0}, \widehat{\mu}_{+}\right)$. The system (4.10) admits a $\left(t_{0}, T\right)$-bifurcation, since the corresponding radius of $\left(t_{0}, T\right)$-attraction satisfies

$$
\lim _{\mu \searrow \mu_{0}} \mathcal{A}_{0}^{\mu}=0
$$

In case the conditions $\left(P 1^{*}\right)$ and $\left(P 2^{*}\right)$ hold, the trivial solution is $\left(t_{0}, T\right)$-repulsive for $\mu \in\left(\widehat{\mu}_{-}, \mu_{0}\right)$ and $\left(t_{0}, T\right)$-attractive for $\mu \in\left(\mu_{0}, \widehat{\mu}_{+}\right)$. The system (4.10) admits a $\left(t_{0}, T\right)$-bifurcation, since the corresponding radius of $\left(t_{0}, T\right)$-repulsion satisfies

$$
\lim _{\mu \nearrow \mu_{0}} \mathcal{R}_{0}^{\mu}=0
$$

The proof of the theorem is similar to that of Theorem 4.1.

\section{An example}

In this section we give an example illustrating our theoretical results by means of simulations. Consider the following system with $I:=[0,10]$ and impulse moments $\theta_{i}=1,2, \ldots, 9$,

$$
\begin{aligned}
\dot{x} & =\left(6 \mu+2.5 \mu \sin \left(t^{3} / 4\right)\right) x-\left(4 \mu+3.5 \mu \sin \left(t^{5} / 3\right)+2\right) x^{2}+\left(\mu+0.5 \mu \cos ^{2}\left(t^{3}\right)\right) x^{3}, \\
\left.\Delta x\right|_{t=i} & =(1.5 i \mu+5 \mu) x-(2 i \mu+5 \mu+3) x^{2}+i \mu x^{3} .
\end{aligned}
$$

One can verify that this system satisfies the conditions of Theorem 4.1. Simulation results reveal that all solutions starting in the neighborhood of the origin converge to the origin if $\mu<0$, whereas for $\mu>0$ all solutions starting in the neighborhood of the origin diverge from the origin. 


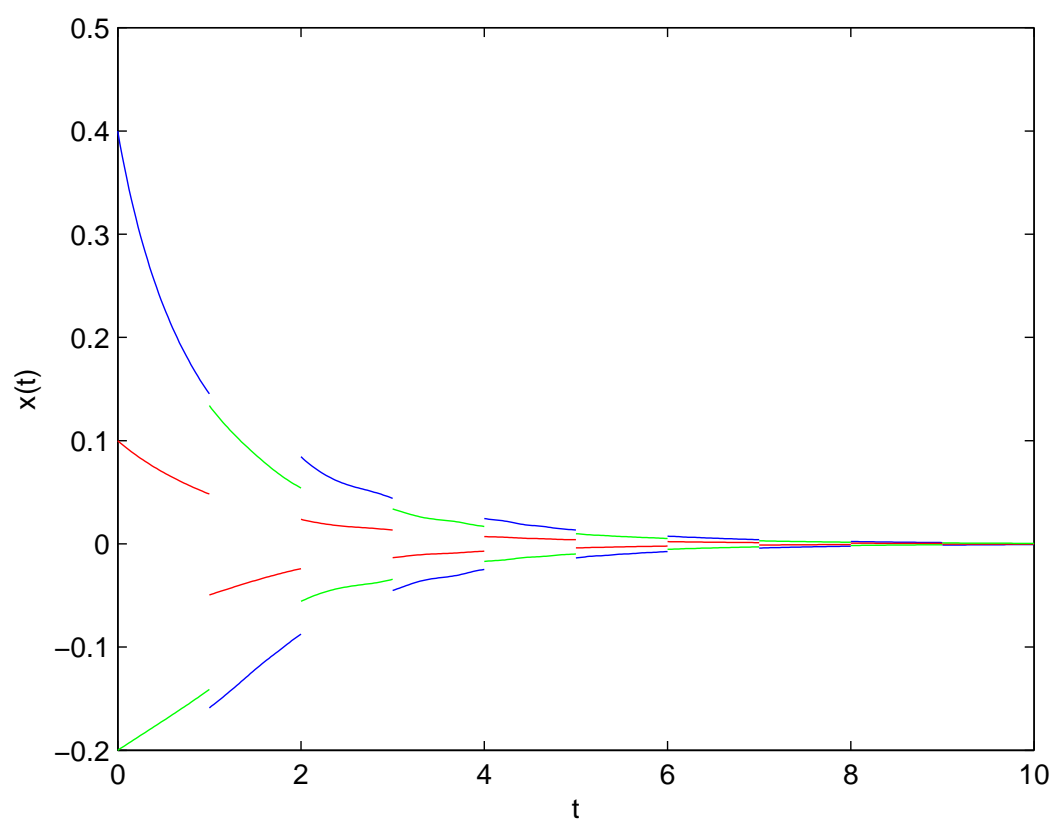

Figure 5.1: Asymptotic behavior of the solution for $\mu=-0.1$, where each color represents a solution corresponding to a different initial value.

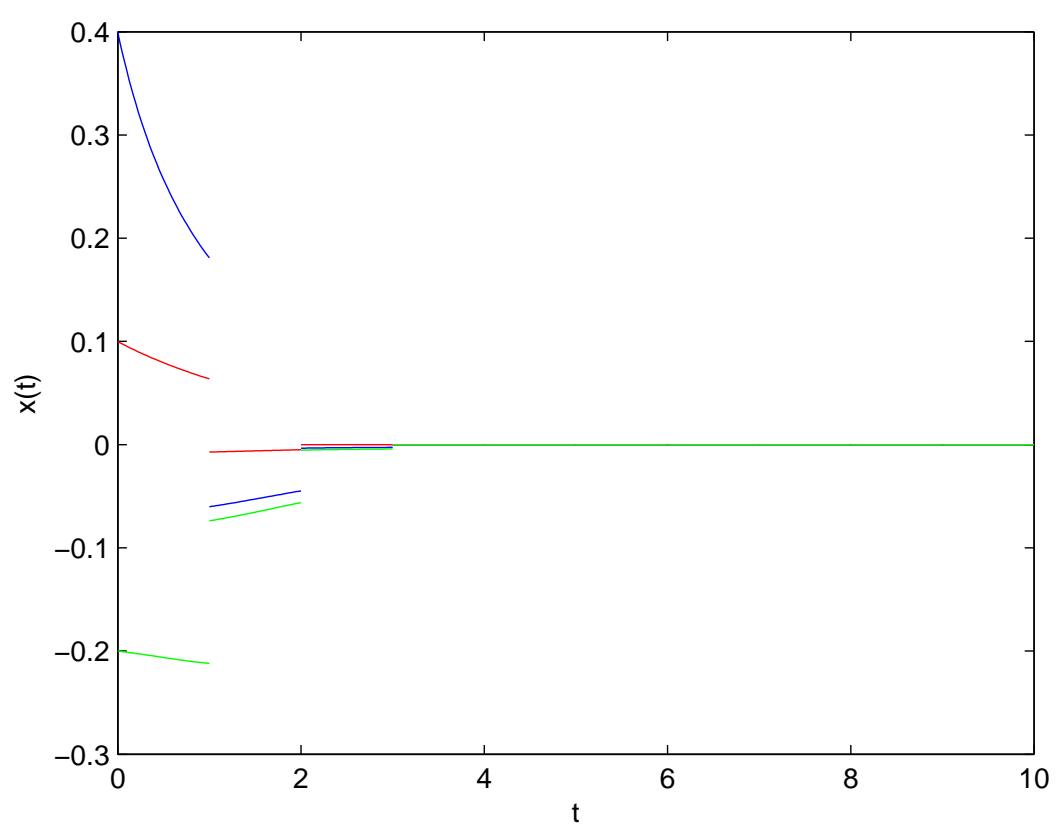

Figure 5.2: Asymptotic behavior of the solution for $\mu=-0.05$, where each color represents a solution corresponding to a different initial value. 


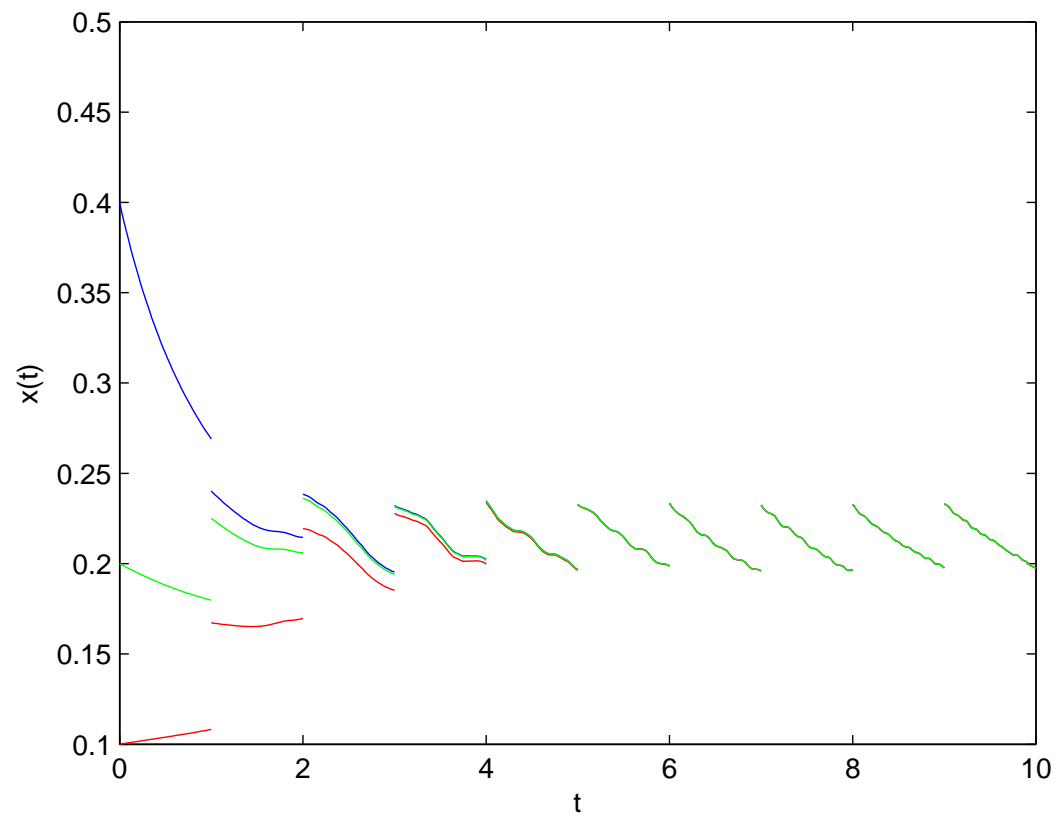

Figure 5.3: Asymptotic behavior of the solution for $\mu=0.05$, where each color represents a solution corresponding to a different initial value.

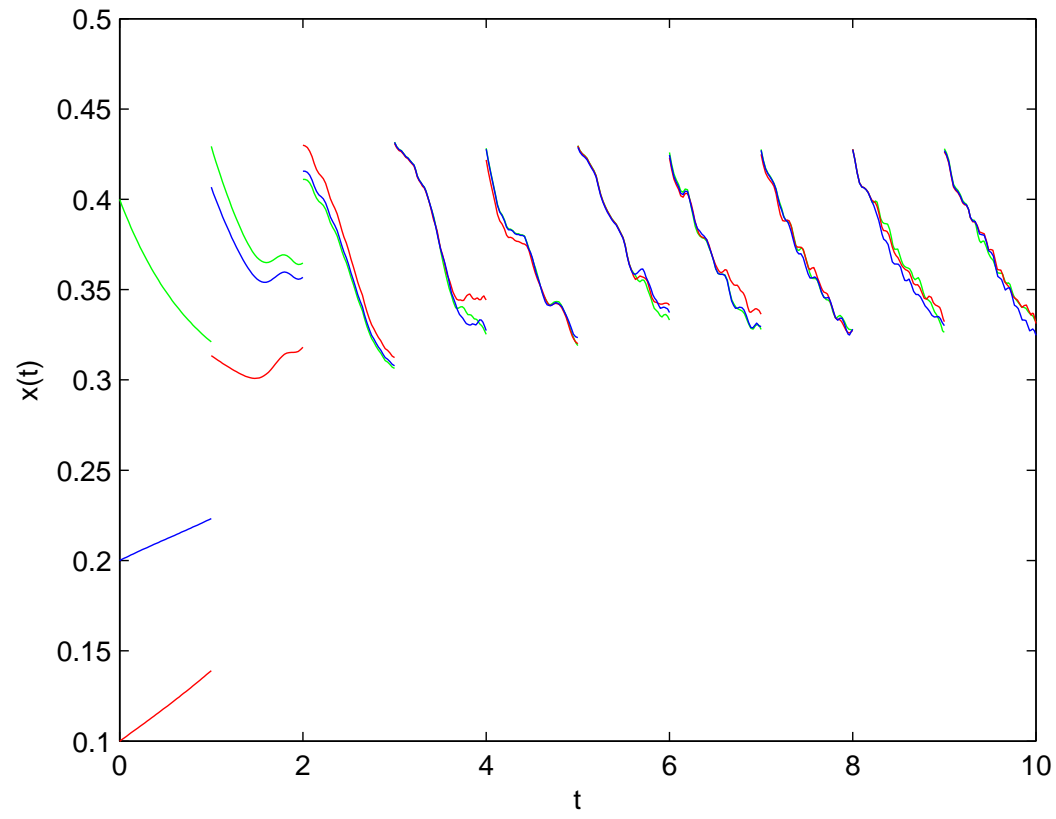

Figure 5.4: Asymptotic behavior of the solution for $\mu=0.1$, where each color represents a solution corresponding to a different initial value.

From the simulation results, it is seen that the trivial solution is $(0,10)$-attractive for $\mu \in$ $(-0.1,0)$ and $(0,10)$-repulsive for $\mu \in(0,0.1)$. Moreover, $\lim _{\mu \nearrow_{0}} \mathcal{A}_{0}^{\mu}=0$ and $\lim _{\mu \searrow 0} \mathcal{R}_{0}^{\mu}=0$. Thus, the example admits a $(0,10)$-transcritical bifurcation. 


\section{References}

[1] M. Акнмет, Principle of discontinuous dynamical systems, Springer-Verlag, New York, Heidelberg, London, 2010. MR2681108; url

[2] M. U. Акнмет, Perturbations and Hopf bifurcation of the planar discontinuous dynamical system, Nonlinear Anal. 60(2005), No. 1, 163-178. MR2101525; url

[3] M. U. Aкhmet, A. Kashkynbayev, Non-autonomous bifurcation in impulsive systems, Electron. J. Qual. Theory Differ. Equ. 2013, No. 74, 1-23. MR3151720; url

[4] M. U. AKhmet, A. Kashrynbayev, Nonautonomous transcritical and pitchfork bifurcations in impulsive systems, Miskolc Math. Notes 14(2013), No. 3, 737-748. MR3153961

[5] M. U. Akhmet, M. Turan, Bifurcations in a 3D hybrid system, Commun. Appl. Anal. 14(2010), 311-324. MR2757400

[6] B. B. Aldridge, G. Haller, P. K. Sorger, D. A. Laffennburger, Direct Lyapunov exponent analysis enables parametric study of transient signalling governing cell behaviour, Syst. Biol. (IEE Proc.) 153(2006), 425-432. url

[7] D. D. Bainov, P. S. Simeonov, Impulsive differential equations. Asymptotic properties of the solutions, Advances in Mathematics for Applied Sciences, Vol. 28, World Scientific, Singapore, 1995. MR1331144

[8] P. E. Kloeden, Pitchfork and transcritical bifurcation in systems with homogeneous nonlinearities and an almost periodic time coefficients, Comm. Pure Appl. Anal. 3(2004), 161-173. MR2059154; url

[9] P. E. Kloeden, M. Rasmussen, Nonautonomous dynamical systems, AMS, Providence, Rhode Island, 2011. MR2808288; url

[10] V. Lakshmikantham, D. D. Bainov, P. S. Simeonov, Theory of impulsive differential equations, Modern Applied Mathematics, Vol. 6, World Scientific, Singapore, 1982. MR1082551

[11] J. A. Langa, J. C. Robinson, A. Suarez, Stability, instability and bifurcation phenomena in non-autonomous differential equations, Nonlinearity 15(2002), 1-17. MR1901112; url

[12] J. A. Langa, J. C. Robinson, A. Suarez, Bifurcation in non-autonomous scalar equations, J. Differential Equations 221(2006), 1-35. MR2193839; url

[13] T. Peacock, J. Dabiri, Introduction to focus issue: Lagrangian coherent structures, Chaos 20(2010), No. 1, 017501. url

[14] M. Rasmussen, Attractivity and bifurcation for nonautonomous dynamical systems, Lecture Notes in Mathematics, Vol. 1907, Springer-Verlag, Berlin, 2007. MR2327977; url

[15] M. Rasmussen, Nonautonomous bifurcation patterns for one-dimensional differential equations, J. Differential Equations 234(2007), 267-288. MR2298972; url

[16] M. RAsmussen, Finite-time attractivity and bifurcation for nonautonomous differential equations, Differ. Equ. Dyn. Syst. 18(2010), 57-78. MR2670074; url 
[17] K. RateitschaK, O. WolkenhaueR, Thresholds in transient dynamics of signal transduction pathways, J. Theoret. Biol. 264(2010), 334-346. MR2981460; url

[18] A. M. Samoilenko, N. A. Perestyuk, Impulsive differential equations, Nonlinear Science Series A, Vol. 14, World Scientific, Singapore, 1995. MR1355787 